\title{
Signature of elastic turbulence of viscoelastic fluid flow in a single pore throat
}

\author{
Eseosa M. Ekanem $\odot,{ }^{1}$ Steffen Berg $\odot,{ }^{1,3,2}$ Shauvik De $\odot,{ }^{4}$ Ali Fadili, ${ }^{3}$ Tom Bultreys, ${ }^{2,5}$ Maja Rücker, ${ }^{1}$ \\ Jeffrey Southwick, ${ }^{3}$ John Crawshaw $(1),{ }^{1}$ and Paul F. Luckham ${ }^{1, *}$ \\ ${ }^{1}$ Department of Chemical Engineering, Imperial College London SW7 2AZ, United Kingdom \\ ${ }^{2}$ Department of Earth Science and Engineering, Imperial College London SW7 2AZ, United Kingdom \\ ${ }^{3}$ Shell Global Solutions International B.V, 1031HW Amsterdam, The Netherlands \\ ${ }^{4}$ Shell India Markets Private Limited, Karnataka 562149, Bangalore, India \\ ${ }^{5}$ PProGRess UGCT, Department of Geology, Ghent University, 9000 Gent, Belgium
}

(Received 28 September 2019; accepted 23 March 2020; published 23 April 2020)

\begin{abstract}
When a viscoelastic fluid, such as an aqueous polymer solution, flows through a porous medium, the fluid undergoes a repetitive expansion and contraction as it passes from one pore to the next. Above a critical flow rate, the interaction between the viscoelastic nature of the polymer and the pore configuration results in spatial and temporal flow instabilities reminiscent of turbulentlike behavior, even though the Reynolds number $\mathrm{Re} \ll 1$. To investigate whether this is caused by many repeated pore body-pore throat sequences, or simply a consequence of the converging (diverging) nature present in a single pore throat, we performed experiments using anionic hydrolyzed polyacrylamide (HPAM) in a microfluidic flow geometry representing a single pore throat. This allows the viscoelastic fluid to be characterized at increasing flow rates using microparticle image velocimetry in combination with pressure drop measurements. The key finding is that the effect, popularly known as "elastic turbulence," occurs already in a single pore throat geometry. The critical Deborah number at which the transition in rheological flow behavior from pseudoplastic (shear thinning) to dilatant (shear thickening) strongly depends on the ionic strength, the type of cation in the anionic HPAM solution, and the nature of pore configuration. The transition towards the elastic turbulence regime was found to directly correlate with an increase in normal stresses. The topology parameter, $Q_{f}$, computed from the velocity distribution, suggests that the "shear thickening" regime, where much of the elastic turbulence occurs in a single pore throat, is a consequence of viscoelastic normal stresses that cause a complex flow field. This flow field consists of extensional, shear, and rotational features around the constriction, as well as upstream and downstream of the constriction. Furthermore, this elastic turbulence regime, has high-pressure fluctuations, with a power-law decay exponent of up to $|-2.1|$ which is higher than the Kolmogorov value for turbulence of $|-5 / 3|$.
\end{abstract}

DOI: 10.1103/PhysRevE.101.042605

\section{INTRODUCTION}

Many chemical and biological processes involve the flow of viscoelastic fluids such as aqueous polymer solution in complex geometries. Understanding how bulk rheology and a specific flow geometry interact, such that a certain pressure drop-flow rate response is observed, is key to predicting the behavior in different geometries, e.g., different types of porous rock, given a specific bulk rheology. In a more general context, improving the understanding of the rheological response of viscoelastic fluids to certain deformation regimes is important for novel industrial process development and optimization. These processes include synthesis of fiberreinforced plastics with molten polymer, catalytic synthesis, food processing, fluid flow in soils, blood flow in the circulatory system of multicellular organisms, and injection of viscoelastic fluids in petroleum reservoirs for enhanced oil recovery, where oil mobilization is greatly desired and increased drag is undesired. All of these require the tailoring of the flow to achieve a desired flow rate-pressure drop response [1-3].

*Corresponding author: p.luckham01@imperial.ac.uk
In such complex geometries, water-soluble polymer solutions frequently exhibit complex rheology due to a combination of strong extensional and shear flows. A clear separation of the different flow regimes and respective rheological behavior, i.e., shear, extension, and unstable flow, is not easily achieved in practice [4]. This complication arises to an extent because the observation of specific rheological behavior is, in many cases, explicitly dependent on the flow geometry. In turn, that makes it difficult to identify the specific cause in terms of rheology (related to molecular properties of the polymer) and the observed phenomenon in terms of flow field when the geometry is very complex. In natural porous media, the complexity of flow geometry is so great that the underlying rheological cause of the observed flow behavior is difficult to assign. When flowing viscoelastic polymer solutions through porous rock at increasing flow rates we first observe a Newtonian plateau in the viscosity, followed typically by shear-thinning behavior, which are largely identical to bulk rheological measurements in shear rheometers without any porous medium involved [see Fig. 1(a)]. Shear thinning is a very desirable behavior, because in this way one can design, for instance, for oil recovery applications, a polymer flood such that during injection at the wellbore the viscosity is 
(a)

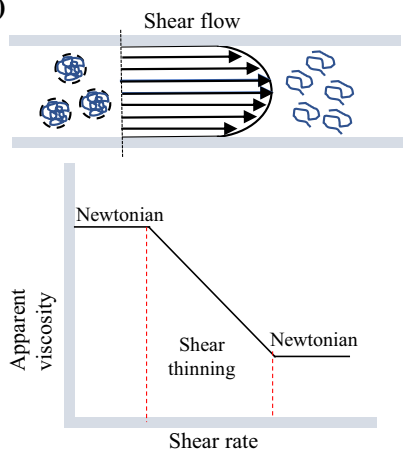

(b)

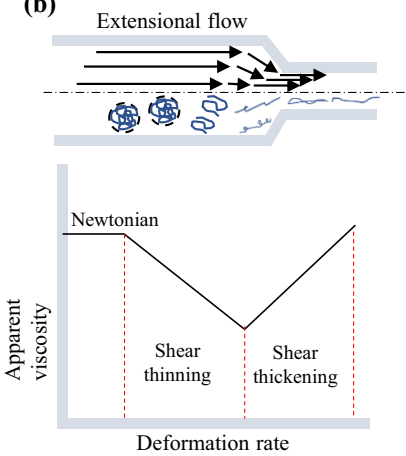

FIG. 1. Schematic illustration of viscoelastic polymer rheology (a) shear flow, such as in rotational rheometers and capillary rheometers showing shear thinning, where a relaxation of the largest polymer chain occurs; (b) extensional flow, such as in porous media with converging (diverging) geometry showing shear thickening, where polymer coils are stretched due to extensional flow.

low, which reduces the required injection pressure, while far away from the wellbore the viscosity is high to increase the efficiency of oil displacement [1]. However, at even higher flow rates we observe an increase in effective viscosity which is undesired as it poses a problem for injectivity [5]. In the past this phenomenon has been interpreted as "shear thickening" $[5,6]$; however, such a regime is not usually observed in simple shear. Only very recently has this phenomenon been attributed to a phenomenon termed "elastic turbulence" which is ultimately caused by viscoelastic behavior of the fluid [7-13].

Understanding the onset of elastic turbulence of viscoelastic polymer solutions in mixed flow systems, i.e., combining both extension and shear, is made more challenging compared to that in a pure shearing flow $[14,15]$ because the observed behavior could be either a consequence of the diverging (converging) geometry, either in one single pore body-pore throat transition, or is the consequence of a collective behavior of many pores in the porous medium where the polymer does not relax before experiencing the next strong deformation.

The flow fields in such repeat unit systems can be imaged; however, the pressure response is an effect of the combination of many pores and throats, meaning in a heterogeneous flow field with many stagnant regions and few dominant flow paths more of the shear-thinning rheology of the non-Newtonian fluid would be emphasized [8,16-18]

The key to understanding this effect is to study these flows in a single pore throat-pore body system. Flexible viscoelastic polymer and polyelectrolyte solutions portray different fluid stresses when either simple shear, or extensional flow, is applied. Hence, in a mixed shear flow where both shear and extensional flow contribute, a combined rheological response becomes evident [19].

The rheological properties of viscoelastic polyelectrolyte solutions depend on concentration, molecular weight, molecular weight distribution, molecular stiffness, and the degree of charge. [20] In this paper, we have considered viscoelastic polymer solutions of hydrolyzed polyacrylamide (HPAM) which is most relevant in oil recovery applications, with concentrations around and above the critical overlap concentra-

tions, $C^{*}$, where the molecules begin to overlap. During shear flow, HPAM shows shear-thinning behavior in a standard rotational rheometer for instance, where the effective viscosity is monotonically decreasing with increasing shear rate in Searle flows [21]. However, within a porous medium, where both extension and shear are experienced, shear thinning also occurs at low shear rates, but is followed by an apparent shear thickening at higher shear rates where the effective viscosity increases with increasing deformation rate (see Fig. 1). This apparent thickening is associated with an enhanced pressure drop and an elastic turbulence occurring at Reynolds number below 1, meaning that the cause of the turbulence is not due to inertial but elastic effects, i.e., a combination of the rheological property of the polymer solution and the specific flow field [12]. Hence, with flexible high molecular weight HPAM the elastic property of the polymer becomes more important and the fluid stresses do not immediately become zero but decay with a relaxation time, $\lambda$, which in turn is dependent on the polymer molecular weight, concentration, and other parameters such as ionic composition. The viscoelastic rheological effects that cause elastic turbulence can also be quantified in terms of the effects of the stress tensor, by the first normal stress difference, $N_{1}$, which is a positive value for non-Newtonian viscoelastic fluids but zero for a Newtonian fluid. In mixed-flow regimes of shear and extension, the normal stresses experienced are either due to shear or to extension and are dependent on the flow history and the degree of extension [19]. The normal stress difference due to shearing is often ascribed to be a result of elasticity, while stresses due to extension are not. Normal stresses due to shear should not be ignored as their contribution may be of the same magnitude as stresses due to extension [19]. De et al. [22] numerically and experimentally studied the contribution of shear and extensional flows to the increased normal stresses of viscoelastic polymer solutions in a porous media, while Haward et al. [14] investigated this contribution experimentally in a cross-slot device and they both found a large contribution of viscoelastic normal stresses in shear flow at high flow rates where elastic turbulence exists $[23,24]$.

The flow behavior of these polymer solutions changes in a nonuniform microchannel, as was demonstrated by Clarke et al. [8], with a transition in flow regime from viscous dominated to unsteady flow as the flow velocity increased. During this unsteady flow, a high power-law exponent greater than $|-5 / 3|$ was also shown in the works of Kawale et al. [3], De et al. [25] and Bonn et al. [9], where the spatial temporal effects of the fluid may become evident and a distribution of stresses occur within the flow caused by normal stresses, due to the fluid's elasticity [26].

Therefore, the key to a more complete understanding of the cause of elastic turbulence comes through the observation of the actual flow fields and the corresponding pressure response. Therefore, in this paper, we use the lowest-level complexity model system available to obtain a better understanding of the flow regimes and rheological effects of viscoelastic solutions in a single hyperbolic contraction channel that also comes with spatially resolved pressure sensors across the contraction. We investigate the rheology based on the pressure drop and flow rate within the channel. The flow device has been modified for optical access allowing direct visualization 
TABLE I. Description and properties of each solution.

\begin{tabular}{lcccccccc}
\hline \hline Solution & Polymer conc. $(\mathrm{ppm})$ & Salt & $C_{\text {salt }}(\mathrm{ppm})$ & $I(\mathrm{mM})^{\mathrm{b}}$ & $\lambda(\mathrm{s})$ & $\eta_{0}(\mathrm{Pas})$ & $\mathrm{De}$ & $\mathrm{Re}$ \\
\hline $\mathrm{Gly}: \mathrm{H}_{2} \mathrm{O}$ & $84^{\mathrm{a}}$ & & & & & $0.06^{\mathrm{c}}$ & & $10^{-2}-10$ \\
$\mathrm{HPAM}-\mathrm{DI}$ & 150 & & & & 10 & 0.45 & $10^{-1}-18$ & $10^{-3}-10^{-1}$ \\
$\mathrm{HPAM}-\mathrm{Na}$ & 2000 & $\mathrm{NaCl}$ & 5000 & 86 & 4 & 0.59 & $10^{-1}-10$ & $10^{-3}-10^{-1}$ \\
HPAM-Ca & 6000 & $\mathrm{CaCl}_{2}$ & 5000 & 90 & 1.4 & 0.35 & $10^{-2}-7$ & $10^{-3}-0.4$ \\
\hline \hline
\end{tabular}

${ }^{\mathrm{a}}$ Concentration of glycerol in wt. \%.

${ }^{\mathrm{b}}$ Ionic strength of salt in millimoles.

${ }^{\mathrm{c} N e w t o n i a n}$ viscosity $(\mu)$ for gly: $\mathrm{H}_{2} \mathrm{O}$ (84:16 wt. \%).

of the flow field based on the particle image velocimetry technique (PIV). We find, for instance, that a critical De at which apparent thickening occurs changes with the presence of $\mathrm{Na}^{+}$and $\mathrm{Ca}^{2+}$ ions, confirming the results of Howe et al. [6] and Seright et al. [27]. The results also demonstrate that elastic turbulence already occurs in a single pore throat with no flow history. We establish this from the imaged flow field and from the scaling exponent in the power spectral density of the pressure fluctuations. Our work also goes beyond the established literature in that we also compare the rheology of the flow for solutions with different ionic strength to the imaged flow field and we compute the flow topology parameter to decouple the shear and extensional contribution to the observed normal stresses.

\section{FLUID SAMPLE CHARACTERIZATION}

In this study we use partially hydrolyzed polyacrylamide polymer (HPAM; obtained from SNF Floerger, FP3630) with a molecular weight $\left(M_{w}\right)$ between 18 and $20 \mathrm{MDa}$, which is a very common system for studying elastic turbulence. The polymer was supplied as a white granular powder with approximately $30 \%$ degree of hydrolysis. In order to vary the degree of the elastic contribution in the polymer rheology, and also reflect realistic conditions for applications, three solutions were prepared by dissolving the polymer powder in deionized water (HPAM-DI) and brine [ $\mathrm{NaCl}$ (HPAM$\mathrm{Na}$ ) and $\mathrm{CaCl}_{2}$ (HPAM-Ca)] at different concentrations [see Table I]. $\mathrm{Na}^{+}$and $\mathrm{Ca}^{2+}$ ions were added for two reasons: firstly, these ions are generally present in reservoir brines where HPAM is commonly used in enhanced oil recovery; secondly, the ions screen the charge on the HPAM making the configuration of the polymer less rigid and more random coil-like in nature, influencing the degree of elastic response in the rheological behavior. The DI water and brines used were filtered and an oxygen scavenger (containing $15 \%$ isopropanol, $7.5 \%$ thiourea, and $77.5 \%$ water) at $20 \%$ of the polymer concentration was added before polymer powder was introduced to prevent oxidation of the polymers. All the solutions were stirred for about $48 \mathrm{~h}$ to ensure complete dissolution. The polymer solutions were then filtered through a $2-\mu \mathrm{m}$ filter under a pressure of 5 bar to remove any microgels. The filtration ratio obtained was close to 1 . The viscosity curve of each solution in Fig. 2(a) was measured using a Thermo-Fisher Haake MARS rotational rheometer fitted with a double-gap concentric cylinder geometry with shear rates spanning between 0.01 and $1500 \mathrm{~s}^{-1}$. To assess the potential for secondary flows, or Taylor instabilities at high shear rates, the Taylor number was estimated to be 15 , indicating that no instabilities are present (see Eqs. (S1) and (S2) in the Supplemental Material provided [28]) [29,30]. The data gathered are summarized in Table I.

The concentrations of the three anionic HPAM solutions were adjusted to give similar zero-shear rate viscosities with the highest polymer concentration required for solutions containing $\mathrm{CaCl}_{2}$. It is important to note that the relaxation times of these solutions are different because their polymer
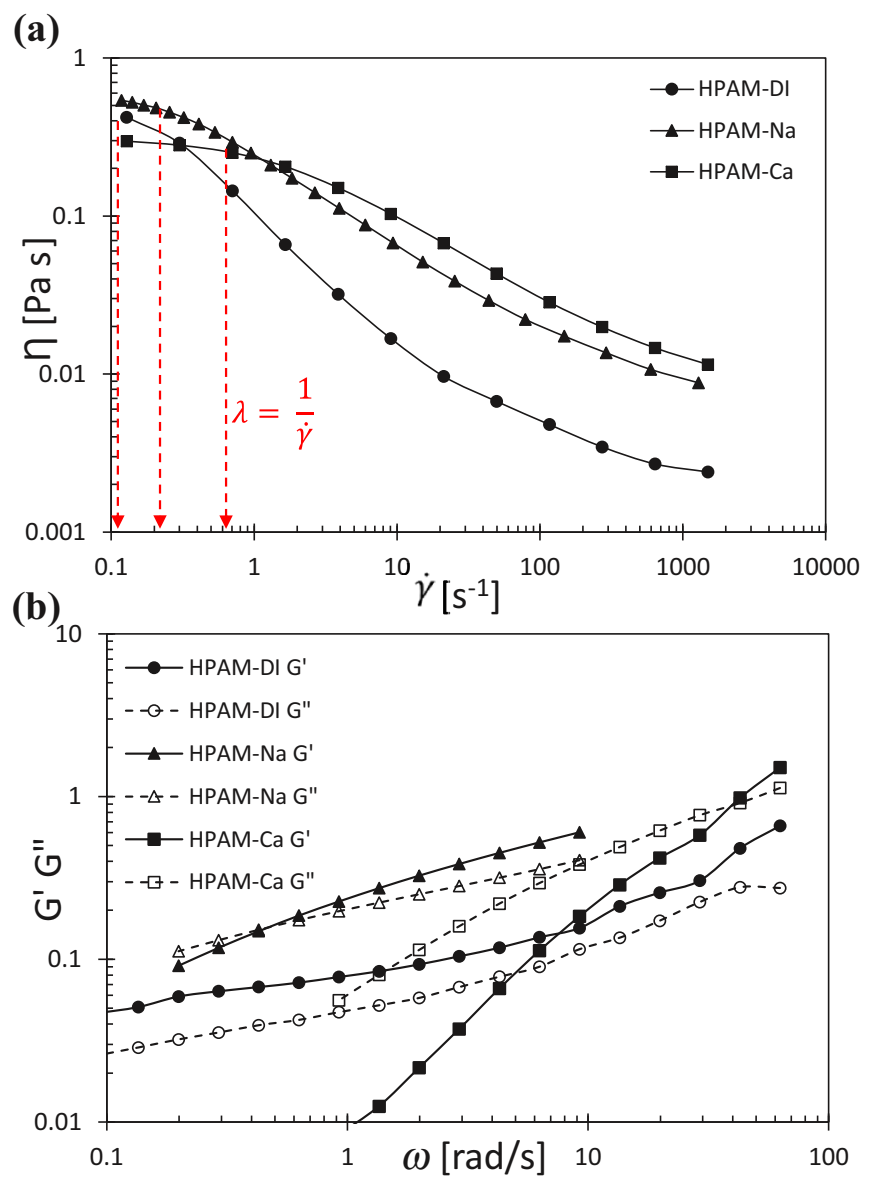

FIG. 2. (a) Shear rheology of all fluids carried out using the double-gap rheometer. $\lambda$ represents the relaxation time obtained from the inverse of the shear rate at the point of shear thinning. (b) Oscillatory shear data of the viscoelastic fluids as a function of the angular frequency showing the crossover point of $G^{\prime}$ (elastic modulus) and $G^{\prime \prime}$ (loss modulus) for HPAM-Na and HPAM-Ca and no crossover for HPAM-DI. 
(a)

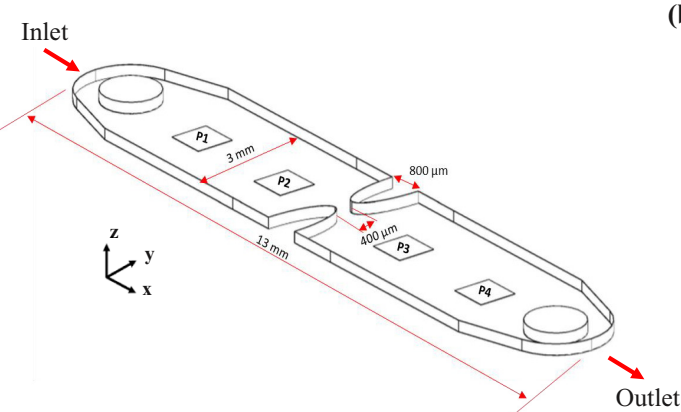

(b)

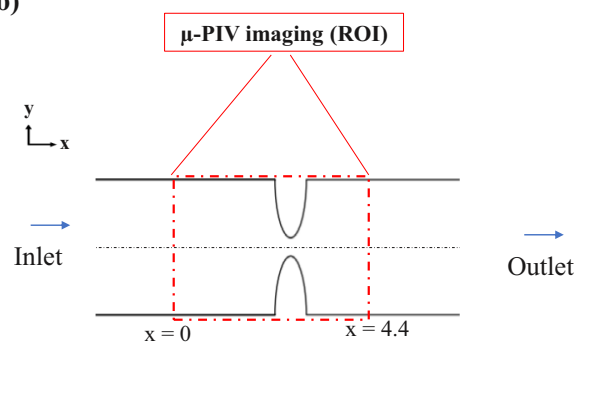

FIG. 3. (a) Schematic description of a pseudo-3D microfluidic channel, extensional viscometer rheometer on a chip, $P_{1}-P_{4}$ are the MEMS pressure sensors. Depth of channel, $h=0.2 \mathrm{~mm}$, contraction length, $l_{c}=0.8 \mathrm{~mm}$, contraction width, $w_{c}=0.4 \mathrm{~mm}$, upstream width, $w_{u}=$ $3 \mathrm{~mm}$ and total channel length, $L=13 \mathrm{~mm}$. (b) Region of interest for the micro-PIV experiment is also shown from $x=0$ to $x=4.4 \mathrm{~mm}$ of the total channel length and contraction point of the channel is at $2.5 \mathrm{~mm}$. A large portion of the observation is located upstream of the contraction in order to capture more of the fluid features upstream (adapted from Ober et al. [42])

concentration and molecular structure varies. In the absence of salt, anionic HPAM-DI is more expanded due to the longranged repulsion between the $\mathrm{COO}^{-}$groups of HPAM chain [31]. The $\mathrm{Ca}^{2+}$ ions and $\mathrm{Na}^{+}$ions in solution can either bridge between these groups, or screen the charges. In either case the effect would be to collapse the chains such that the polymer concentration required to attain similar zero-shear rate viscosity increases (calcium ions being divalent are more effective at this than the monovalent sodium ions) $[32,33]$. The relaxation time, $\lambda$ (seconds) of each polymer solution was determined from Fig. 2(a) by taking an inverse of the shear-rate value where the transition from Newtonian to shear thinning occurs. We note that these polymer solutions show different relaxation mechanisms characterized by different relaxation times, and the relaxation time obtained here represents the longest relaxation time. In Fig. 2(b), the results of oscillatory shear experiments are presented, and the elastic, $G^{\prime}$ and loss, $G^{\prime \prime}$ modulus are shown as a function of frequency. We observed that all solutions showed a crossover point in $G^{\prime \prime}$ and $G^{\prime}$ except HPAM-DI, due to its very low concentration and therefore low elasticity [34]. However, it is difficult to assess the order of increasing elasticity in the shear rheometer; this is better understood in the extensional viscometer rheometer on a chip (eVROC) in situ rheology measurements presented later.

Flow curves, such as the ones displayed for the fluids used in this study in Fig. 2(a), show three regimes: (1) The zero-shear rate viscosity regime with a Newtonian viscosity plateau, where the shear rates tend towards zero, and the polymer chains are said to be in a coiled state without any shear disturbances. (2) As the shear rate increases, there is transition to a shear-thinning region in which the longest relaxation time of the unperturbed molecule is obtained [35,36] and (3) the infinite shear viscosity regime occurring at high shear rates.

The shear-thinning curves for each solution show a different power-law slope of $-0.39,-0.49$, and -0.59 for HPAM-Ca, HPAM-Na, and HPAM-DI, respectively, because the HPAM conformation has been modified [32,33,37,38]. This result shows that the effect of ionic components on HPAM causes a reduction in its viscosity $[39,40]$. Schroeder et al. [41] showed that large rotational fluid geometries do not affect the polymer molecular conformation, but when a strong extensional component dominates, it causes the poly- mer chains to reorient, stretch, and change its conformation. This is because in a converging (diverging) geometry such as in a porous media where extensional components become apparent, a deviation from simple shear flow in a rheometer occurs, as in the case of Fig. 1(b), and hence the effects in a rheometer cannot be translated directly to the porous media.

\section{EXPERIMENTAL PROCEDURE}

\section{A. Single pore throat micromodel}

Two identical single pore throat micromodels with a planar hyperbolic contraction at the middle of the channel were obtained from RheoSense (San Ramon CA, USA). Both devices were fabricated with the standard wet-etching method using Pyrex glass. One of the micromodels (eVROC) has four microelectromechanical (MEMS) pressure sensors located at the base of the lower channel plate and the entire micromodel is enclosed in an opaque black box. Hence, visualization of the fluid flow in this micromodel was not possible. The second micromodel, identical to the first but transparent and without the MEMS pressure sensors, was used for visualization. A schematic of the micromodel is shown in Fig. 3(a) and in Fig. 3(b) along with the following dimensions: channel length, $L=13 \mathrm{~mm}$, upstream width $w_{u}=3 \mathrm{~mm}$, contraction width, $w_{c}=0.4 \mathrm{~mm}$, contraction length, $l_{c}=0.8 \mathrm{~mm}$, and height of the channel, $h=0.2 \mathrm{~mm}$. The region of interest (ROI) imaged during the micro-PIV experiments is depicted in Fig. 3(b). The hyperbolic contraction is located at the center of the channel. This hyperbolic contraction geometry gives a crosssectional area which varies inversely with the axial position and hence, in principle, a constant nominal extensional rate can be imposed [42].

The average velocity profile $u_{\mathrm{av}(x)}$ along the flow direction is computed as $u_{\mathrm{av}(x)}=\frac{Q}{\mathrm{hw}(x)}$, where $Q$, is the flow rate, $w(x)$ is the cross-section width at any $x$ position, and $h$ is the depth of the channel. The apparent extensional rate, $\dot{\varepsilon}_{a}$ is estimated using

$$
\dot{\varepsilon}_{a}=\frac{Q}{l_{c} h}\left(\frac{1}{w_{c}}-\frac{1}{w_{u}}\right),
$$


with $l_{c}$ the length of the contraction. The relative apparent viscosity, $\eta_{r}$ is computed as the ratio

$$
\eta_{r}=\frac{\Delta P_{c} / u_{\mathrm{av}}(\text { viscoelastic })}{\Delta P_{c} / u_{\mathrm{av}}(\text { Newtonian })},
$$

where $\Delta P_{c}$ is an interpolation of the pressure drop across the channel constriction from $P_{1}$ to $P_{4}$ and $u_{\mathrm{av}}=\frac{Q}{\mathrm{hw}_{u}}$ is the average velocity upstream of the flow channel [43]. The Deborah number, De, which is the ratio of the fluid's relaxation time, $\lambda$, to the resident time, $\tau_{r}$, in the control volume is computed as [13]

$$
\mathrm{De}=\frac{\lambda}{\tau_{r}}=\frac{\lambda u_{\mathrm{av}}}{L},
$$

where $u_{\mathrm{av}}$ is the average velocity across the channel and $L$ is the channel length. The first normal stress difference in this geometry is estimated using the method in Ober et al. [42] as follows:

$$
N_{1}=\frac{\Delta P_{c}}{\varepsilon_{H}},
$$

where, $\varepsilon_{H}$, is the maximum logarithmic strain (Hencky strain) of the polymer solution occurring in the contraction channel. For this specific geometry, the Hencky strain derivation can be found in Ober et al. [42].

The Reynolds number, Re, which represents the ratio of inertia to viscous forces, was defined as

$$
\operatorname{Re}=\frac{\rho d_{h} u_{\mathrm{avc}}}{\eta_{0}},
$$

where $d_{h}=\frac{2 \mathrm{hw}_{c}}{\left(h+w_{c}\right)}$ is the hydraulic diameter at the contraction of the channel, $\rho$ is the fluid density, and $\eta_{0}$ is the zero-shear viscosity of the fluid. The velocity $u_{\mathrm{avc}}$ is the average velocity in the contraction obtained from $\frac{Q}{\mathrm{hw}_{c}}$ (instead of $u_{c}=l_{c} \dot{\varepsilon}_{a}$, which is a characteristic velocity describing the change in the average velocity from device inlet to the contraction inlet as proposed by Ober et al. [42]). However, since Re is below unity using either velocity, inertia is not seen to play a significant role in the flow of the polymer solution through the microchannel.

\section{B. Microparticle image velocimetry}

The characterization of the velocity field was carried out using microparticle image velocimetry ( $\mu$-PIV) in Fig. 4 $[44,45]$. The imaging was carried out using an inverted microscope (Leica DMi8), comprising an objective lens of $2.5 \times$ magnification (Leica HC PL Fluotar 2.5×/0.07 numerical aperture objective) and a Thorlabs plano-convex round cylindrical lens $(f=250 \mathrm{~mm})$ enclosed in a $2.5 \times$ camera adapter (Leica 10441675). The PIV camera was a Phantom Lab340 $(1440 \times 2560$ pixels $)$ charge-coupled device camera with a frame rate of 800 frames per second.

The initial exposure time was set at $550 \mu$ s and then reduced as the fluid flow rate was increased to avoid the formation of long streaks in the images while ensuring that the particle displacement between successive frames was optimal for the cross correlation. The solutions were seeded with 2.08- $\mu \mathrm{m}$ polystyrene microspheres obtained from microParticles $\mathrm{GmbH}$ with a concentration of $0.5 \%$ w/v. To acquire

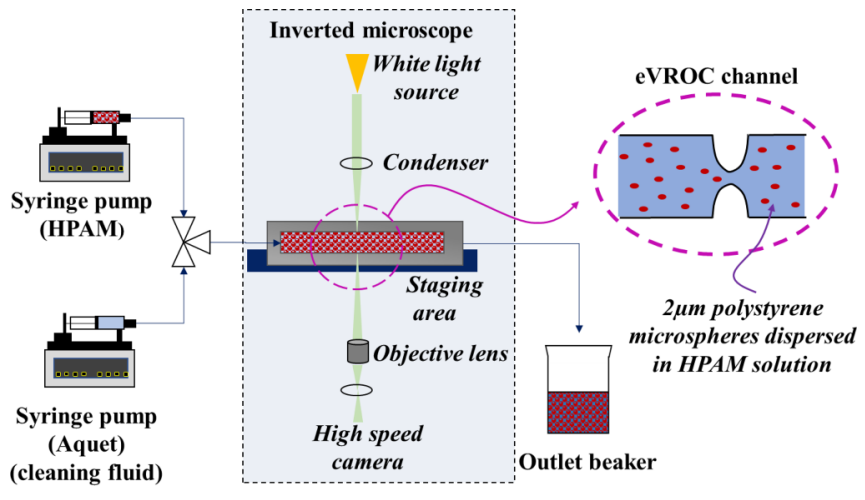

FIG. 4. Schematic of the microparticle image velocimetry technique.

images at a constant $x-y$ plane located at the midpoint of the channel depth ( $z$ axis), focal points were acquired with the aid of supplier markings located at the top and bottom of the channel. The mid $z$-axis points at $100 \mu \mathrm{m}$ were estimated from the average of the focal-point values. The cross-correlation calculation and the velocity distribution were obtained by postprocessing the images using PIVLAB, an open-source MATLAB-based software [46].

For fluid injection, two 10-mL gas-tight Hamilton syringes were used, one containing the HPAM solution to be measured and the other containing an Aquet solution, a cleaning fluid. A three-way valve at the inlet allowed the injection of either solution into the channel and prevented introducing air bubbles while switching between flows. A long length of tubing free from any constriction connected the channel outflow to the collection vial. The tube ensured a constant flow across the microfluidic device and prevented any downstream perturbations that may have resulted in back flow.

\section{Flow characterization in the eVROC}

In the following, we use the flow field obtained by $\mu$-PIV for a Newtonian fluid consisting of $84 \mathrm{wt}$ \% glycerol in water to characterize the flow geometry and establish a Newtonian reference. The reference solution $\left(\right.$ gly: $\left.\mathrm{H}_{2} \mathrm{O}\right)$ was imaged in the single contraction channel by the $\mu$-PIV technique at flow rates of 25,50 , and $100 \mu \mathrm{l} / \mathrm{min}$ which corresponds to Reynolds number $0.04,0.07$, and 0.15 , respectively. The images were taken at the center plane of the channel along the $z$-axis depth. Figure 5(a) shows the velocity distribution of the entire flow domain for the ROI. Lateral velocity profiles of the $x$-component velocities were extracted upstream of the contraction channel and plotted in Fig. 5(b) for flow rates at $50 \mu \mathrm{l} / \mathrm{min}$. A symmetric flow pattern is observed upstream and downstream of the contraction channel. As expected, the maximum velocities occurred along the centerline axis of the channel and reduces progressively towards the channel walls. The maximum velocity is approximately $1.9 \mathrm{~mm} / \mathrm{s}$ at the center of the contraction. The axial velocity profiles along the centerline axis in the $x$ direction at different flow rates are shown in Fig. 4(c). The fluid accelerates towards the contraction channel with peak velocities at the contraction entrance and decelerates immediately on exiting the contraction. 


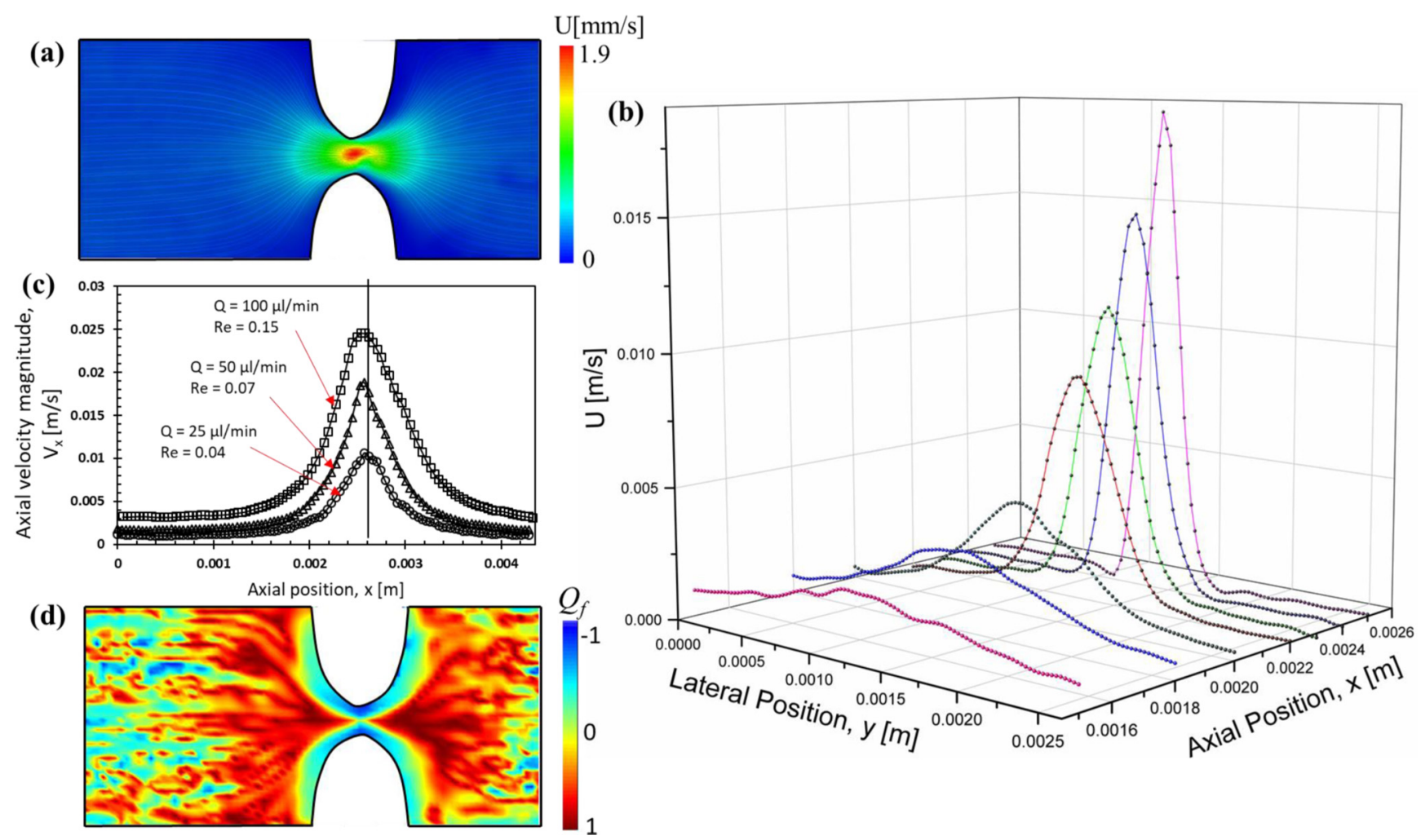

FIG. 5. Characterization of gly: $\mathrm{H}_{2} \mathrm{O}(84: 16$ wt. \%) (a) velocity magnitude field within the measured flow domain for $Q=50 \mu \mathrm{l} / \mathrm{min}$, $\operatorname{Re}=0.07$ and (b) lateral velocity profile at the same flowrate in a (c) axial velocity profile for $Q=25,50$, and $100 \mu 1 /$ min corresponding to $\operatorname{Re}=0.04,0.07$, and 0.15 , respectively (d) Flow topology characterization at $Q=50 \mu \mathrm{l} / \mathrm{min}$

To differentiate between flow dominated by shear and flow dominated by extensional kinematics, we use the flow topology parameter $Q_{f}$, following Haward et al. [14] and De et al. [22] as

$$
Q_{f}=\frac{|\dot{\gamma}|-|\Omega|}{|\dot{\gamma}|+|\Omega|}
$$

where $|\dot{\gamma}|$ and $|\Omega|$ are the magnitudes of the deformation rate and vorticity tensors, respectively. Those values are estimated from the local velocity component in each grid point within the ROI flow domain. For $Q_{f}=1$, the flow is typically dominated by extensional kinematics. For $Q_{f}=-1$, the flow is purely shearing and when $Q_{f}=0$, the flow is essentially rotational. [14,47-49].

Figure 5(d) shows the flow topology for gly: $\mathrm{H}_{2} \mathrm{O}$ at a flow rate of $50 \mu \mathrm{l} / \mathrm{min}$. The flow domain shows more extensional kinematics immediately upstream and downstream of the contraction and along the centerline axis of the channel, indicating that an extensional rate is imposed across the channel and the initial extension effects occur three orders of the contraction throat length upstream of the contraction channel [42]. However, at the contraction walls a more shear and rotational kinematics of the flow is observed.

The macroscopic quantities of the Newtonian fluid estimated from the eVROC such as the viscosity was constant while $N_{1}$ increased linearly [50] (see Figs. S1(a) and S1(b) in the Supplemental Material [28]). This is because the geometry is not a simple shear flow but a mixed flow of shear and extension; hence, the enhancement of the normal stresses is small compared to what is experienced by the non-Newtonian HPAM solutions.

\section{RESULTS AND DISCUSSION}

\section{A. Flow resistance and normal stresses}

The result of a series of experiments carried out with the eVROC for the relative apparent viscosity, $\eta_{r}$, normal stress, $N_{1}$, and pressure drop as a function of De are shown in Figs. 6(a)-6(c). $\eta_{r}$ and De were estimated using Eqs. (2) and (3), whereas De increases the apparent viscosity changes. At low De, the flow curves exhibit strong shear thinning except for HPAM-Ca, which shows a limited degree of shear thinning. The behavior appears to be caused by divalent $\mathrm{Ca}^{2+}$ ions, which screen the polymer negatively charged molecular chain of the $\mathrm{COO}^{-}$group causing a collapse of the coil [27]. This screening effect is reduced for HPAM-Na because $\mathrm{Na}^{+}$ monovalent ion does not screen the charge so well and it is not observed in HPAM-DI.

In Fig. 6(a), at a critical De $\sim 0.5$, the flow resistance changes for HPAM-Na and HPAM-Ca, where a deviation in $\eta_{r}$ begins and is related to a transition due to the fluid's elasticity. In addition, the critical onset of shear thickening in Fig. 6(a) is similar to the onset of increased normal stresses, $N_{1}$, in Fig. 6(b) and varies across all polymer solutions. For HPAM-Ca it occurs at $\mathrm{De}_{c} \sim 1, \mathrm{De}_{c} \sim 3$ for HPAM-Na, and $\mathrm{De}_{c} \sim 5$ for HPAM-DI. We also note that the degree of shear thickening is relatively less for HPAM-DI than the other fluids. 

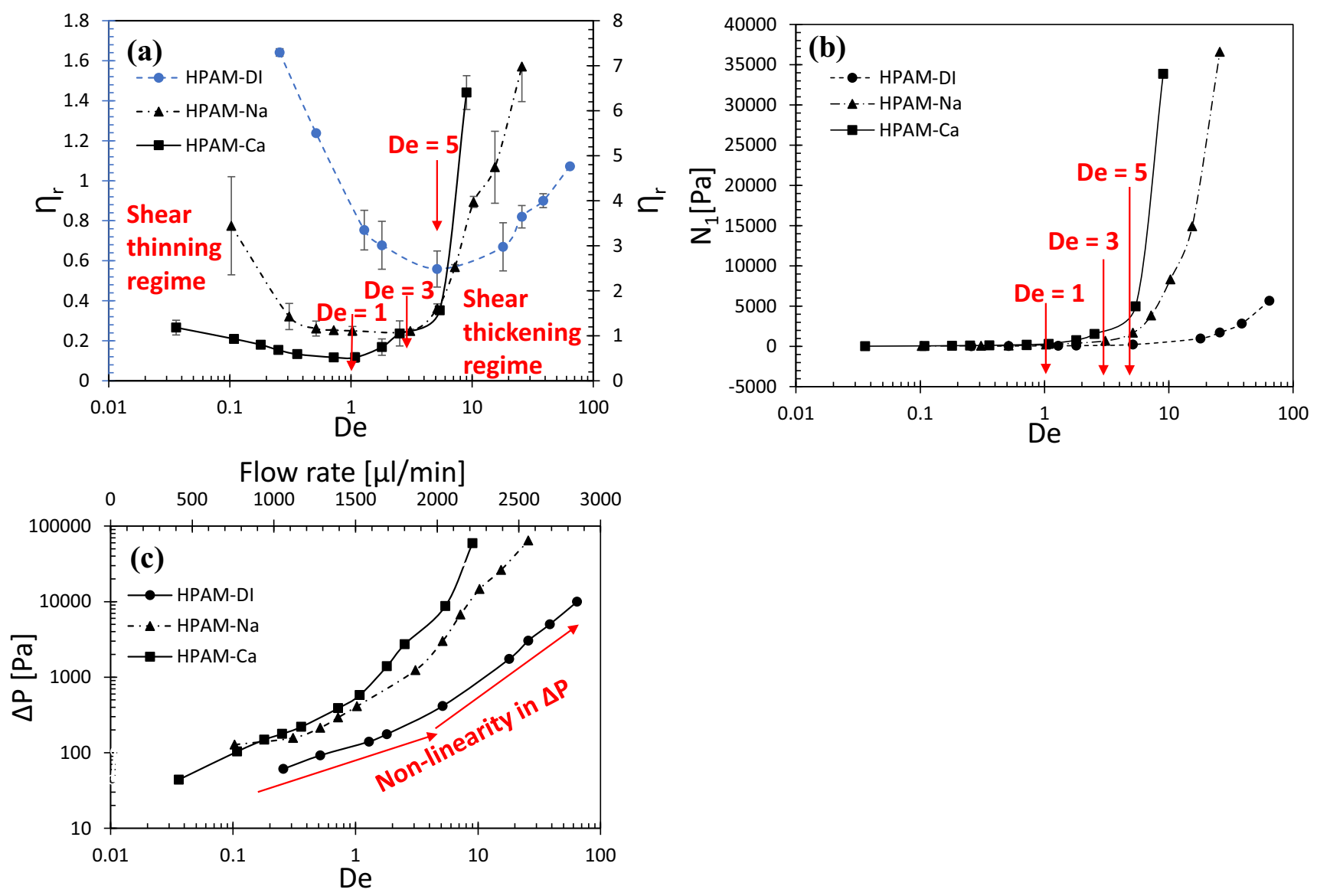

FIG. 6. (a) Semilog plot of relative apparent viscosity as a function of the dimensionless Deborah number (De) for HPAM-DI, HPAM-Na, and HPAM-Ca; the error bars are a standard deviation from three experimental runs. (b) Normal stress difference as a function of De. The critical onset of shear thickening in graph a occurs at the same De as the critical onset of increased normal stresses in graph b. (c) Pressure drop in the constriction as a function of De with the equivalent flow rate in the secondary horizontal axis, showing a deviation from linear to nonlinearity. A symmetric increase in $N_{1}$ with HPAM-DI $<$ HPAM-Na $<$ HPAM-Ca.

In Fig. 6(a), we observe a systematic trend in the rheological behavior of HPAM with different onsets and magnitude of shear thickening which is related to the difference in conformation of the polymer and demonstrates more of the links between the polymer molecular interaction and bulk rheology. In the presence of high salinity, as in the case of HPAM-Ca, where there is a stronger interaction between divalent $\mathrm{Ca}^{2+}$ and anionic polymer chains, the polymer chains exist as rather compact random coils and so more energy would be required to uncoil the structure in order to flow through the channel pore throat compared to HPAM-Na and HPAM-DI [27]. In the work of De Gennes, polymer stretching occurs at a certain critical flow rate where the polymer exists as a coiled state before the critical value and then becomes stretched after the critical value, due to the hydrodynamic forces exerted on the polymer $[35,51]$. Consequently, the nature of the coil to stretched state is highly dependent on the size of the contraction geometry, which induces drag on the flow in the stretched state relative to the coiled state and in turn sharpens the coil-stretch transition. However, we establish here that by changing the type of cation different magnitudes of the shear thickening can occur without varying the contraction width.

The first normal stress difference, $N_{1}$ obtained from Eq. (4) is plotted in Fig. 6(b). High $N_{1}$ indicates strong elastic stresses where the Weissenberg effect is greatly enhanced, and we see an increased $N_{1}$ occurring at the start of the shear thickening where $\operatorname{Re}<1$ (see $\mathrm{Re}$ range in Table I). This experimental rheological result agrees with the simulation of $3 \mathrm{D}$ porous media flows by De et al. [22], where the normal stresses were greatest at regions of higher flow resistance, and Bodiguel et al. [52]. Other studies that have shown increased flow resistance in porous structures include Kawale et al, [3] Mitchell et al. [11], Clarke and coworkers [11], De et al. [36], Minale [53], Paduano et al. [54], and Zamani et al. [55]. In addition, previous works of Keller and Odell for viscoelastic polymer solution have also found increased birefringence after a critical De, until a saturation point where polymer chains become fully stretched and break in half, causing degradation $[56,57]$. However, in the experiments performed here, these saturation point flow rates were not reached due to experimental limitations.

\section{B. Imaging the flow field in the single pore throat by PIV}

In Fig. 7, the velocity streamlines obtained from the $\mu$-PIV measurements for all fluids are shown at different De. The aim here is to characterize the flow field in more detail and establish a connection of how the flow-field geometry 


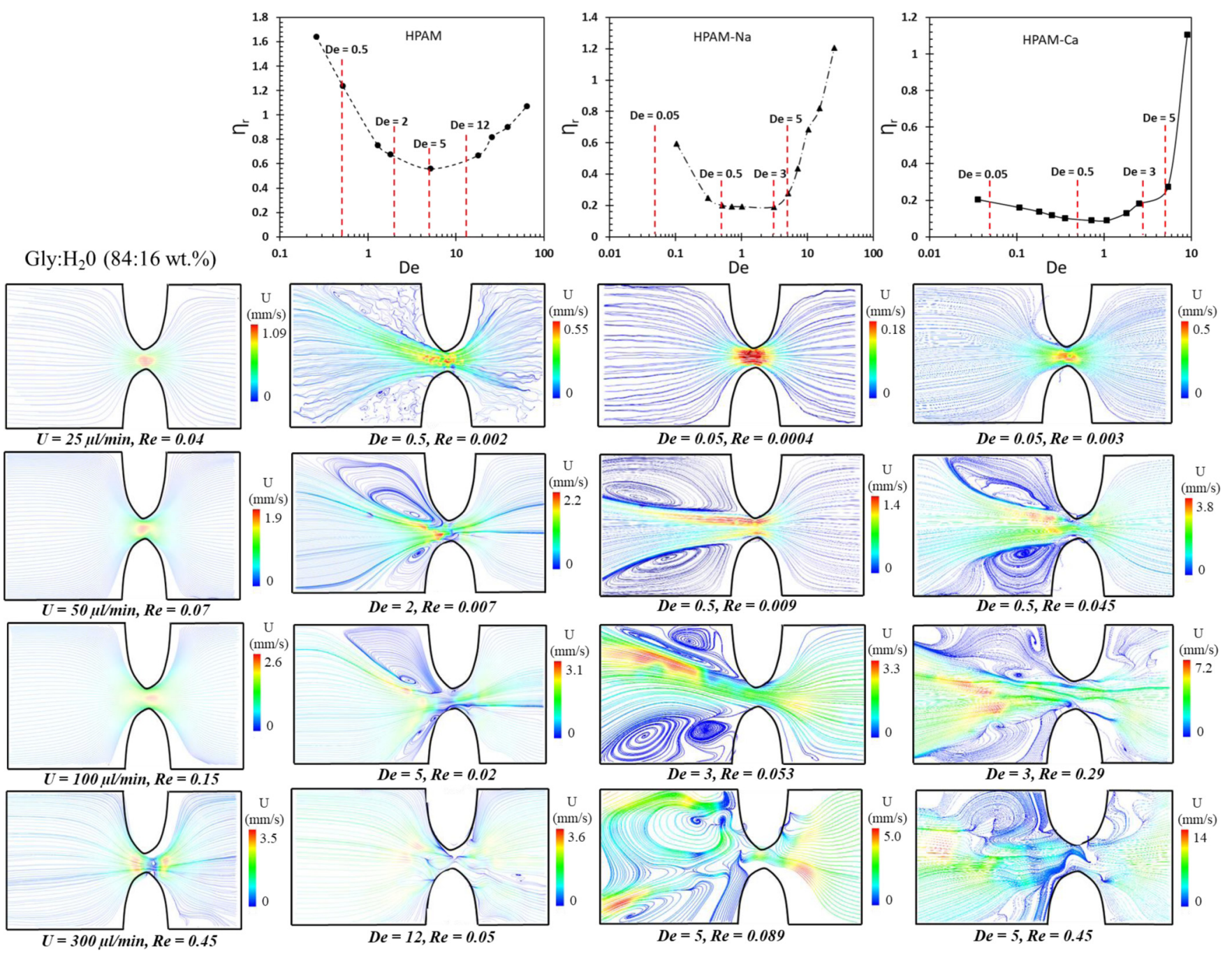

FIG. 7. A comparison of the flow resistance curves and the velocity streamline in $\mathrm{mm} / \mathrm{s}$ of two consecutive image pairs (average time between image pairs $=1.25 \mathrm{~ms}$ ) with increasing De and Re. Flow resistance curves and velocity streamlines are shown for all polymer solutions and compared with Newtonian fluid (gly: $\left.\mathrm{H}_{2} \mathrm{O}\right)$. Symmetry flow is observed for Newtonian fluids and asymmetry flows for non-Newtonian polymer solutions at high De.

in conjunction with the rheological properties of the bulk fluid causes the elastic turbulence. In order to do this, we inspect the evolution of the vortices within the flow geometry. We first observe that in the Newtonian case $\left(\mathrm{gly}: \mathrm{H}_{2} \mathrm{O}\right)$, the velocity streamlines are symmetric compared to the polymer solutions. For HPAM-DI at De $<5$, where the rheology is shear thinning, the streamlines are characterized by large circulating vortices at the corner entrance of the contraction which later disappear at De $>5$ to an unstable flow occurring principally at the contraction entrance. For HPAM-Na and HPAM-Ca, symmetric velocity streamlines similar to gly: $\mathrm{H}_{2} \mathrm{O}$ occur at low $\mathrm{De} \sim 0.05$. At this flow rate, the polymer chains which exist in a random-coil configuration are not disturbed by hydrodynamic drag forces. At De $\sim 0.5$, the configuration of the velocity streamline changes, where large circulating vortices are seen at the corner entrance of the contraction and fast-moving velocity streamlines at the center towards the contraction inlet. When compared to the rheology, this is where shear thinning is being experienced. As the flow progresses to higher De $>3$ for HPAM-Na and De $>1$ for HPAM-Ca, unstable flow occurs where the elastic nature of the polymer solution becomes more pronounced and total symmetry is lost. At this point, the rheology is within the shear-thickening regime and the nature of turbulence is known as the so-called elastic turbulence $[3,12,22,25]$.

This observation is clearly shown in the movies provided via the Supplemental Material [28] (S1-S4) for HPAM-Na and (S5-S8) for HPAM-Ca. Hence, based on the movies and images presented in Fig. 7, we infer that with the polymer solution used here, elastic turbulence already occurs in a single pore throat without any geometrical flow history and the observed vortices are caused by an interplay between the viscoelastic nature of the fluid and the obstruction of the channel at the throat. This causes a distortion in the alignment of the velocity streamlines and the random circulating vortices, thereby creating unsteady flow upstream of the contraction. This unsteady flow observed in the movies, moves spatially and temporally within the channel $[9,10,58,59]$. 


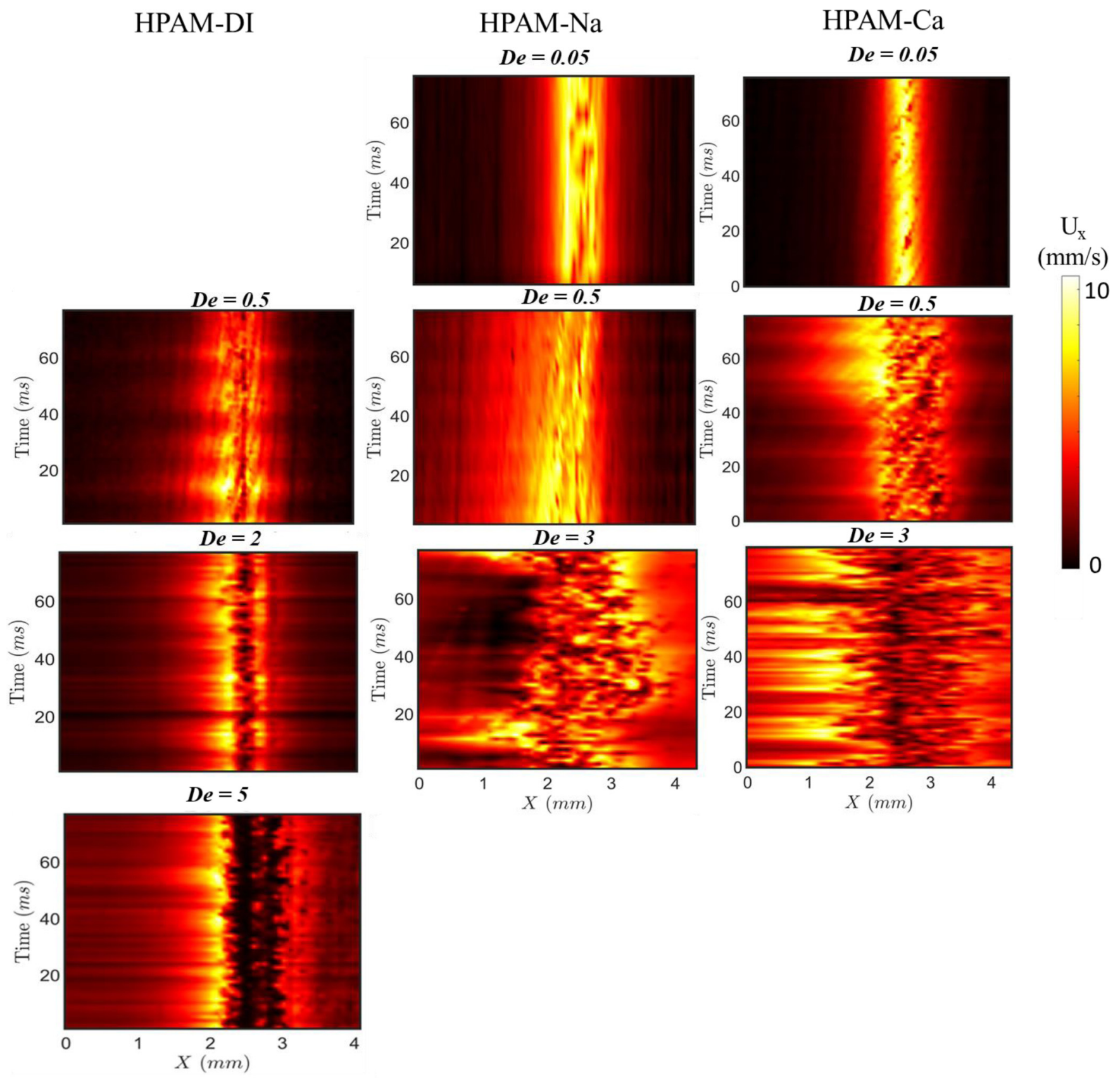

FIG. 8. Time dependence of the velocity fields in $\mathrm{mm} / \mathrm{s}$ for HPAM-DI at De $=0.5,2$, and 5 , HPAM-Na at De $=0.05$, 0.5, and 3, and HPAM-Ca at De $=0.05,0.5$, and 3. The entire observed region is $4.4 \mathrm{~mm}$ and the center of the channel where the throat is located is at $2.5 \mathrm{~mm}$. Space-time plot of the velocity in the $x$ direction at the centerline axis with increasing time (0-75 ms).

\section{Spatial-temporal mapping of the transition from laminar to elastic turbulence}

As can be clearly seen in the videos (S1-S8) presented in the Supplemental Material [28], the streamline images presented in Fig. 7 vary with time. Therefore, in order to capture the spatial-temporal variation of the flow field in the unstable regime, space-time plots for HPAM-DI, HPAM-Na, and HPAM-Ca at different De are shown in Fig. 8. Velocities were taken along the center axis of the flow domain of the ROI and plotted over time. At De $~ 0.05$, HPAM-Na and HPAMCa show a steady and symmetric flow field, where high veloci- ties occur at the contraction and low velocities are experienced upstream and downstream of the contraction without notable spatial-temporal fluctuations. At De $\sim 0.5$, where based on Fig. 6(a) shear-thinning rheology is experienced, all the fluids experience lateral displacement of symmetrical flow. As De increases to around the critical value, increasing from HPAMCa to HPAM-Na to HPAM-DI, more of the unsteady flow is observed upstream of the contraction zone, which increases over time. This long timescale, which coincides with larger spatial length scales, become more important as De increases. Based on the flow field in Fig. 7, this appears to happen because the instability is seeded at the pore throat, such that 
eddies are generated, which are then advected away from the throat. However, the eddies grow in space and eventually begin to decay in the open flow space downstream of the throat as the length- and timescales increase.

Furthermore, due to the unique capabilities of the eVROC, where access to the fluid's pressure field within the channel can be obtained through the four MEMS pressure sensors, the pressure gradient across the contraction channel can be determined.

The pressure drop across the contraction between $P 1$ and $P 3$ as a function of time for HPAM-Na and HPAM-Ca is analyzed and shown in Fig. 9 at different De as the flow moves from steady symmetric to unstable asymmetric flow regime. In Figs. 9(a) and 9(b), the pressure in the low-De region reaches a plateau; however, in the high-De region where elastic effects are larger, a high-pressure fluctuation occurs, indicating that the amplitudes of these fluctuations are increasing with De. Also, the pressure fluctuation in Fig. 9(a) is more unstable compared to Fig. 9(b) in the high-De region. This could be related to the rheology in Fig. 6(a), where HPAM-Ca shows a Newtonian-like behavior of the relative apparent viscosity at low De before a sudden increase in viscosity compared to HPAM-Na. Seright et al. [27] further explains this as a situation whereby more energy is required to unravel the coiled polymer chain bearing calcium ions. Hence, the reason for the relatively small pressure fluctuations at low De and the sudden jump in viscosity and pressuredrop fluctuations for HPAM-Ca in Fig. 6(a) and Fig. 9(b), respectively.

The power spectral density of the pressure fluctuation was computed via a fast Fourier transform of the pressure-drop time series data. Examples for De $=7$ shown in Figs. 9(c) and 9(d) show a power-law decay at high frequencies $[3,10,12,25,60]$. Respective power-law exponents $\beta$ displayed in Fig. 9(e) start from near-zero values at De $<2$, followed by a transition region and reach at De $=7$ exponents, $\beta$ of $|-1.9|$ and $|-2.1|$ for HPAM-Na and HPAM-Ca, respectively. More detailed future studies in a broader variety of systems should clarify whether these fluctuations are truly random or structured and a more detailed analysis with sufficient averaging may be required. Nevertheless, the power-law decay of the pressure-fluctuation spectrum is a clear indication for a flow instability on a broad range of spatial and temporal scale $[3,10,12,25,60]$. Similar power-law decay of pressure fluctuations has also been observed in turbulence of Newtonian fluids, only the exponent observed here is higher than the exponent for Kolmogorov turbulence which has a powerlaw decay of $|5 / 3|$ [25]. Taken together with the fact that the Reynolds number, $\operatorname{Re}<1$, this clearly indicates that the instability observed here is not inertia driven, but indications from both flow field in Fig. 7 and pressure in Fig. 9 in relation to the Deborah number and normal stresses point to an elasticdriven instability.

\section{Flow topology characterization}

In the reasoning that the observed instability is indeed driven by the elastic component in the rheology, we need to be able to relate the dominant flow kinematics with the respective rheology. For that purpose, we characterize the flow regime using a flow topology parameter in order to decouple the effects of extensional and shear kinematics within the flow domain based on Eq. (6), where $Q_{f}$ represents $-1,0$, and +1 corresponding to pure rotational, pure shear, and pure extensional kinematics, respectively.

In Fig. 10(a), we show the topology maps for HPAMNa. At De $=0.05$, the flow topology of the flow domain is similar to gly: $\mathrm{H}_{2} \mathrm{O}$ shown in Fig. 5(d), where most of the flow upstream and downstream is dominated by an extensional kinematic flow, $Q_{f} \sim 1$ (a red color in the figure). As De increases to 0.5 , the extensional flow upstream of the contraction is partially displaced by shear flow. However, increasing the flow to $\mathrm{De} \geqslant 3$, the region upstream of the contraction, where loss of symmetry is experienced, is now being dominated by shear-rotational kinematics, $Q_{f} \sim 0$ and -1 (yellow and blue color in Fig. 10). Similar results are observed for HPAM-Ca in Fig. 10(c). We observe that there is a shift in the flow kinematics from an extensional to a shear-rotational flow field as the fluid flows through the constriction and these observations are similar to the numerical simulations of De et al. [22] for viscoelastic solutions flowing through a random porous medium, where at high De, shear flow dominates over extensional flow. This was also observed experimentally by Haward et al. [14] and agrees with the findings of Groisman and Steinberg [12].

The respective histogram curves in Figs. 10(b) and 10(d) were computed based on the entire ROI, which further demonstrates that the flow is more shear flow dominated at high De with greater peaks around $Q_{f}=0$ and lower peaks at $Q_{f}=1$ for $\mathrm{De}=3$ and 5. Generally, for increasing De, the peak in the histogram shifts from an initial extensional-shear behavior to a shear-rotational kinematic flow behavior. Although, we agree with the works of De et al. [22] and Haward et al. [14], we also take into consideration the importance of the actual stress contribution. For instance, using the stress-strain equation, $\sigma=\eta \dot{\gamma}=\eta \frac{d u_{x}}{d y}$ or $\frac{d u_{x}}{d x}$, depending on the flow direction, to better understand the stress contribution. In the high-De region, where the extensional viscosity is large as in Fig. 6(a), a product of the viscosity and the velocity gradient would lead to a large extensional stress even if the velocity or its velocity gradient is relatively small. However, in a shear flow, where the viscosity is reducing a higher velocity gradient would be required to balance the stresses.

Therefore, looking at the flow topology parameter in Fig. 10, at $\mathrm{De}=0.05$, more of the extensional flow field is seen but it becomes displaced by shear and rotational flow field as De increases. This shift in the flow parameter can be attributed to the large extensional viscosities, which are of the same magnitude as those in the work by Southwick and Manke [61]; this moderates the flow field in such a way that the extensional flow velocity is reduced, and the shear flow velocity is increased with some rotational flow also occurring, which brings about the vortices that are observed. However, this does not necessarily mean that the stresses observed in the high-De region where the so-called elastic turbulence is prominent are due to shear, because taking account of the kinematics of the flow field alone can be misleading. In our opinion, the extensional stresses should also be considered, and in this geometry, we have complex flow that combines both extensional and shear stresses, where the extensional 

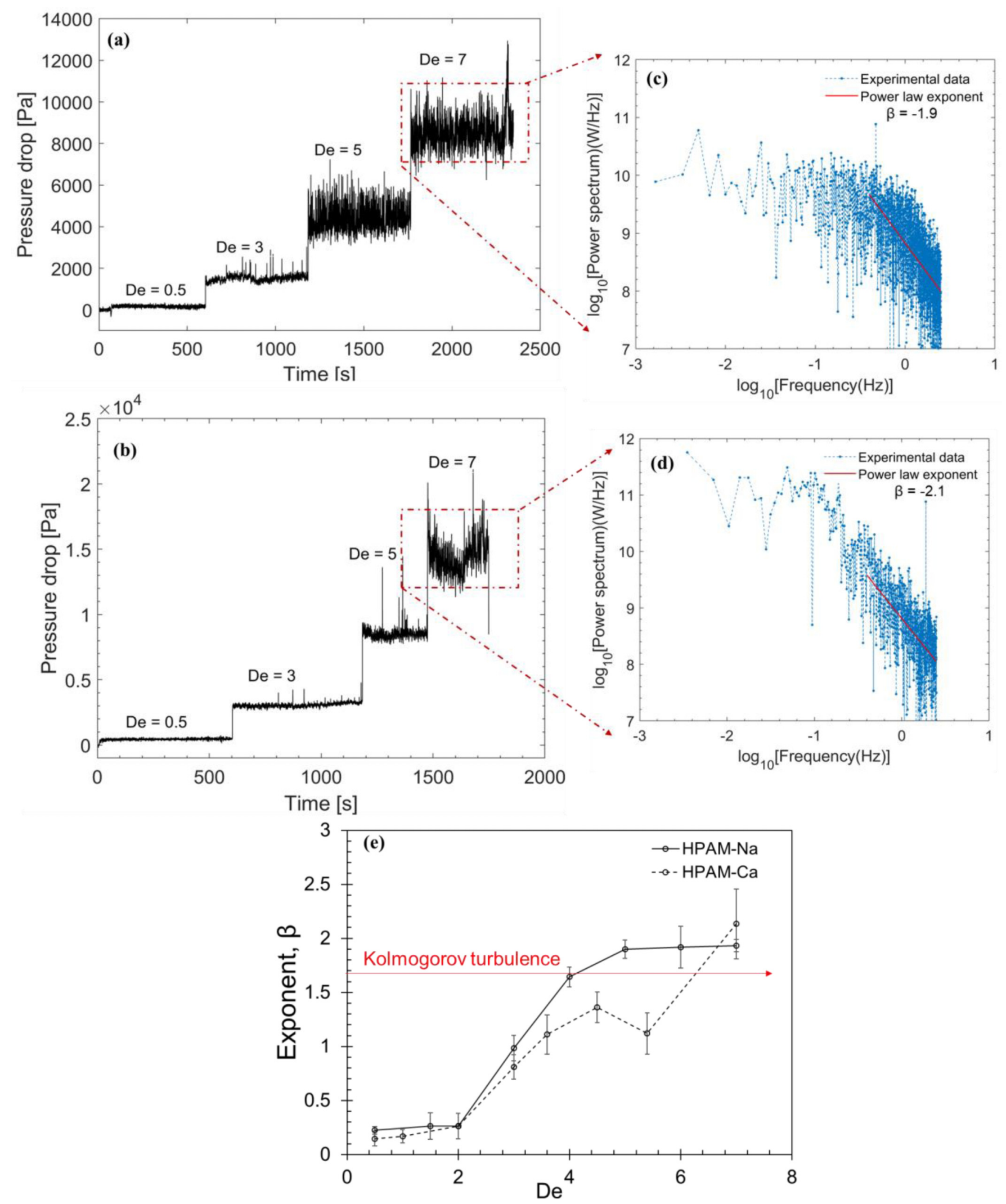

FIG. 9. Pressure drop between $P 1$ and $P 3$ of the MEMS pressure sensor in the eVROC as a function of time for (a) HPAM-Na and (b) HPAM-Ca at different $\mathrm{De}=0.5,3,5$, and 7 indicating the changes in pressure fluctuations. (c), (d) Power spectral density as a function of frequency obtained from the pressure drop-time data plot in (a) and (b) at De $=7$ for both HPAM-Na and HPAM-Ca. The power spectrum is fitted by the power law $P \sim f^{-1.9}$ and $P \sim f^{-2.1}$ for HPAM-Na and HPAM-Ca, respectively, over a frequency range of -0.5 and 1.5 . (e) Plot of the power-law exponent, $\beta$ as a function of De ranging between 0.05 and 7 . The error bars of the exponent were obtained from the standard deviation of three experimental runs. 

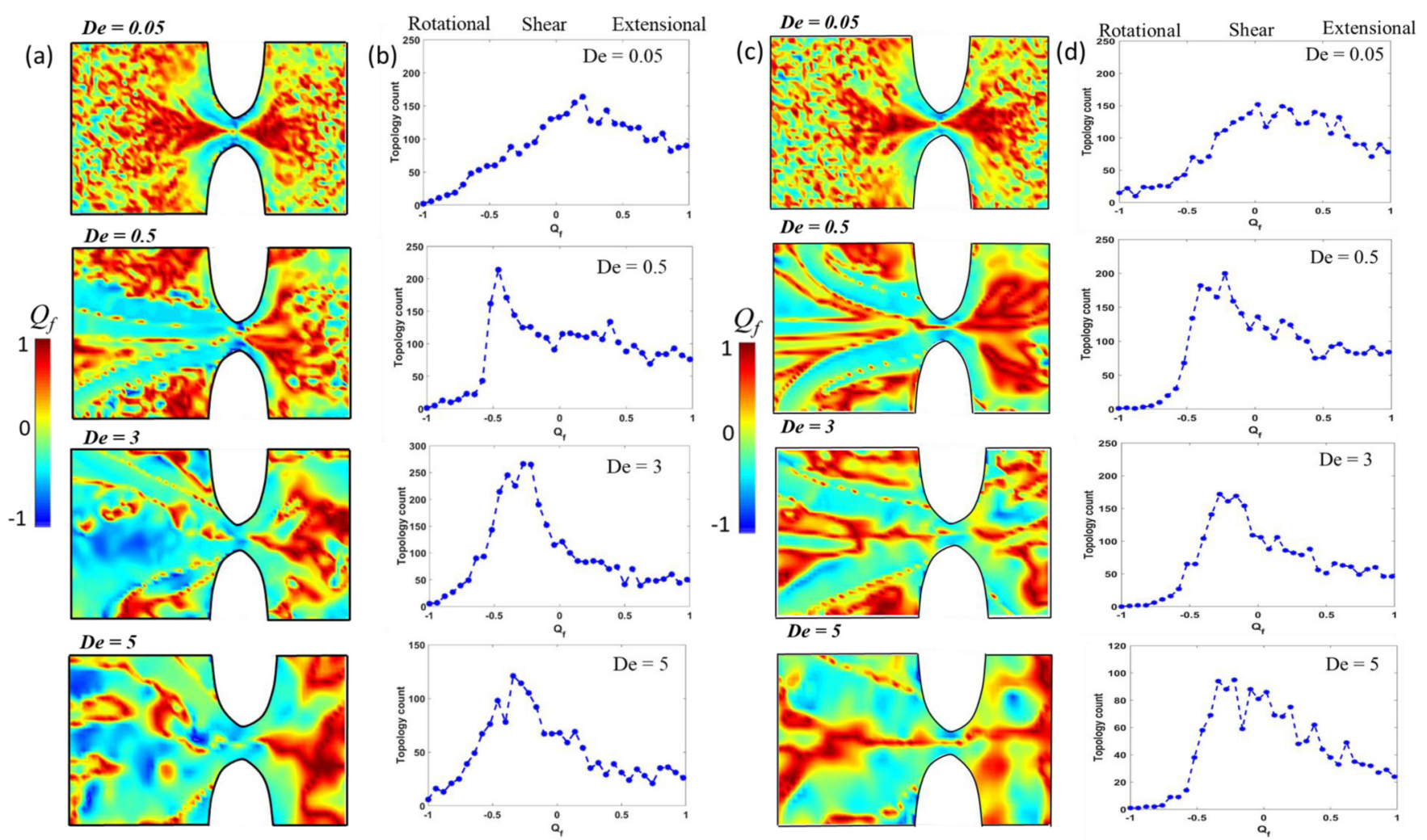

FIG. 10. Flow topology parameter $Q_{f}$ for (a) HPAM-Na and (c) HPAM-Ca at different De numbers $0.05,0.5,3$, and 5 . $Q_{f}$ was resolved from the spatial velocity distribution over time average of two successive frames within the flow domain of the single pore throat geometry. Time between each frame is $1.25 \mathrm{~ms}$. Blue $(-1)$ flow is rotational. Yellow-green ( 0$)$ flow is shear dominated and red $(+1)$ flow is extensionally dominated. Flow topology parameter histogram curves for (b) HPAM-Na and (d) HPAM-Ca obtained from the whole ROI for each De listed in (a) and (c) respectively.

stresses dominate. We note that, in Fig. 6(b), the normal stress $N_{1}$ increases in the high-De region by a factor between 10 and 100 depending on the fluid. This further links to the observations of Keller and Odell [56] where in a contraction geometry (at deformation rates exceeding those possible here), the polymer molecules break in half, which supports our hypothesis that the stresses are extensional dominated. On the other hand, for a Newtonian fluid where both shear and extensional viscosity are constant (at fixed ratio) this phenomenon does not occur, as evident from the glycerol case in Fig. 7.

Therefore, combining the observations and implications from the spatial-temporal analysis in Fig. 8, the flow topology parameter in Fig. 10 and scaling of the pressure fluctuation spectrum in Fig. 9, we present in Fig. 11 a plot of the normalized normal stress $N_{1}$, a measure of the elastic stresses, against the Deborah number, De. It is in effect a "pseudophase diagram" of these flow transition shown in a dimensionless parameter space for the evolution of the instabilities based on the viscoelastic polymer solutions used in this paper.

We compare the behavior below and above the critical onset of the Deborah number where there is a transition from a steady Newtonian-shear-thinning regime to an asymmetric flow regime. Elastic stresses, as proposed in the elastic turbulence model by Groisman and Steinberg [12], are a possible cause for the instability. Indeed, in the low elastic region just before the transition regime, a steady symmetric flow occurs, which in the transition regime becomes a steady asymmetric flow and at higher De becomes an unsteady asymmetric flow, characterized by spatially and temporally displacing vortices. In this region, the elasticity of the polymer

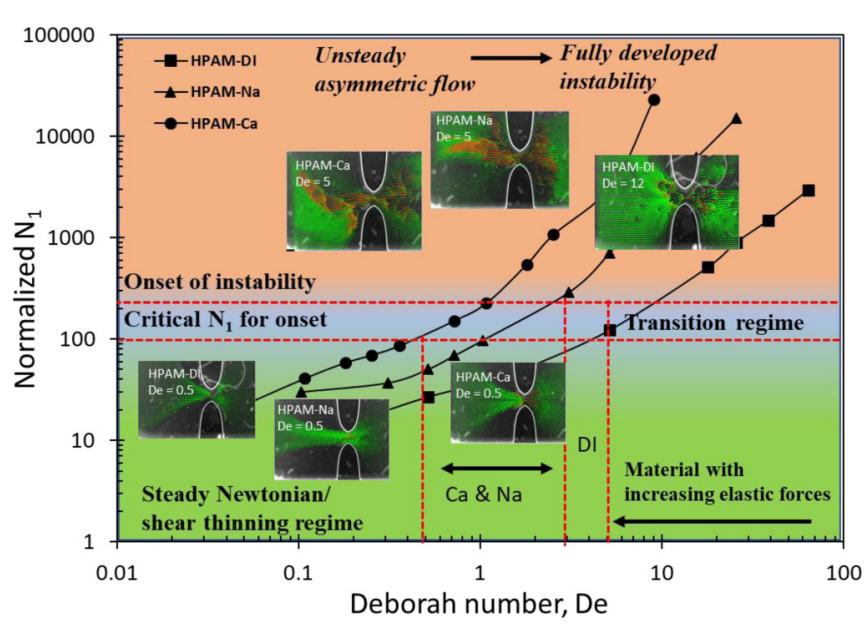

FIG. 11. Normalized normal stress, $N_{1}$ versus Deborah number, De showing the flow transition for all fluids, (HPAM-DI (HPAM + water), HPAM-Na (HPAM $+\mathrm{NaCl})$ and HPAM-Ca (HPAM + $\mathrm{CaCl}_{2}$ )) from steady shear thinning to steady asymmetric and unsteady asymmetric flow where instability is fully developed. 
becomes increasingly significant and the instabilities become fully developed. The flow behavior observed in our work can be related to the previous work of Rodd et al. [62] in an abrupt contraction geometry and Alves and Poole [63], where the elastic turbulence is occurring at a critical De. In order to fully close this loop, i.e., provide evidence that the instability is elastically driven, we compare in Fig. 11 the onset of the instability in terms of De for the three different fluids, which is based on the ionic composition. It clearly shows a systematically increasing trend in terms of the elastic contribution in the rheology. The normalized $N_{1}$, representing the elastic forces, as a function of the Deborah number fully represents the different transition regimes. We observe in this dimensionless parameter space that when the flow is steady, the normalized $N_{1}$ is low but as $N_{1}$ increases the flow becomes unsteady, due to the increasing elasticity of the fluids. We confirm that the onset of the instability for HPAM-Ca, HPAM$\mathrm{Na}$, and HPAM-DI in terms of De varies systematically with the degree of normal stress, which provides further evidence that the instability is indeed elastically driven with a complex flow field induced by a combination of both extension and shear stresses.

\section{CONCLUSIONS}

In this study, we have investigated the flow behavior of viscoelastic polymer solutions composed of water-soluble polyacrylamide (HPAM) in deionized water and in the presence of salt $\left(\mathrm{NaCl}\right.$ and $\left.\mathrm{CaCl}_{2}\right)$ through a well-defined single pore throat microfluidic channel (eVROC). Characterization of the different HPAM solutions was carried out with a rotational rheometer for bulk rheological properties. However, since the key interest is in porous media, we considered a converging (diverging) flow geometry representative of a single pore throat in a porous media system for probing the in situ rheology. Apparent viscosity and the normal stress difference were determined in the eVROC device by a four-point pressure measurement and the flow field was imaged by $\mu$-PIV.
The key finding is that the phenomenon of elastic turbulence that has been reported previously in porous media with many repeat units is reproduced here by a single pore throat. This opened the route to studying the coupling of the fluid rheology and flow field within the converging (diverging) geometry. The very detailed study on this single pore throat has enabled a much more detailed relationship between flow field and pressure response to be developed, and also established an understanding of how the flow geometry triggers the instability. Hence, we find that fluctuations within the channel geometry, which are related to the elastic turbulence, are induced by the sudden contraction of the geometry together with the material properties, causing the polymer chain to reorient and stretch. This affects the viscosity, and the magnitude and direction of the streamlines become broken and/or distorted. Therefore, the key learning of this study is that the elastic turbulence effect is not caused by a (non)relaxation time phenomenon of polymer solution flow between adjacent pore throats although this may add to the complexity, but that the root cause already occurs in the single pore throat. The normal stress, $N_{1}$ and the apparent extensional viscosity observed in the single pore throat, increased at the same De. The pressure fluctuations in the unstable flow regime which were analyzed based on the Fourier transform showed a power-law decay exponent as high as $|-2.1|$ considerably above the range of the Kolmogorov turbulence. The $\mu$-PIV images and the power-law exponent show that at high De and high frequency, respectively, large velocity fluctuations occur over a broad range of spatial and temporal scales. These fluctuations which were characterized by the flow topology parameter confirms that the observed elastic turbulence is due to viscoelastic normal stresses resulting in a complex flow field, which consists of shear and extensional components and also rotational elements leading to elastic turbulence.

\section{ACKNOWLEDGMENTS}

The authors gratefully acknowledge the funding of the Shell Digital Rocks Programme.
[1] L. W. Lake, Enhanced Oil Recovery (Prentice-Hall, Englewood Cliffs, NJ, 1996), Vol 1, p.600.

[2] J. F. Steffe, Rheological Methods In Food Process Engineering (Freeman Press, East Lansing, MI, 1996), Vol 2.

[3] D. Kawale, E. E. Marques, P. L. J. J. Zitha, M. T. Kreutzer, W. R. Rossen, and P. E. Boukany, Soft Matter 13, 765 (2017).

[4] C. E. Wagner and G. H. McKinley, J. Non-Newtonian Fluid Mech. 233, 133 (2016).

[5] A. Thomas, Essentials of Polymer Flooding Techniques (WileyBlackwell, 2019).

[6] A. M. Howe, A. Clarke, and D. Giernalczyk, Soft Matter 11, 6419 (2015).

[7] D. Kawale, Ph.D. thesis, Technische Universiteit Delft, 2017.

[8] A. Clarke, A. M. Howe, J. Mitchell, J. Staniland, and L. A. Hawkes, SPE J. 21, 0675 (2016).

[9] D. Bonn, F. Ingremeau, Y. Amarouchene, and H. Kellay, Phys. Rev. E 84, 045301(R) (2011).
[10] P. C. Sousa, F. T. Pinho, and M. A. Alves, Soft Matter 14, 1344 (2018).

[11] J. Mitchell, K. Lyons, A. M. Howe, and A. Clarke, Soft Matter 12, 460 (2016).

[12] A. Groisman and V. Steinberg, Nature (London) 405, 53 (2000).

[13] A. Varshney and V. Steinberg, Nat. Commun. 10, 652 (2019).

[14] S. J. Haward, G. H. Mckinley, and A. Q. Shen, Sci. Rep. 6, 1 (2016).

[15] H. A. Barnes, J. F. Hutton, and F. R. S. Walters, An Introduction to Rheology (Elsevier, Amsterdam, 1993).

[16] S. Berg and J. van Wunnik, Transp. Porous Media 117, 229 (2017).

[17] D. Wang, J. Cheng, Q. Yang, G. Wenchao, L. Qun, and F. Chen, in Proceedings of the SPE Annual Technical Conference and Exhibition, Texas, 2000 (Society of Petroleum Engineers, 2000).

[18] J. A. Odell and S. J. Haward, Rheol. Acta 47, 129 (2008).

[19] D. F. James, J. Non-Newtonian Fluid Mech. 232, 33 (2016). 
[20] R. G. Larson, Rheol. Acta 31, 213 (1992).

[21] A. Thota Radhakrishnan, J. van Lier, and F. Clemens, Water 10, 1287 (2018).

[22] S. De, J. A. M. Kuipers, E. A. J. F. Peters, and J. T. Padding, Soft Matter 13, 9138 (2017).

[23] S. De, P. Krishnan, J. van der Schaaf, J. A. M. Kuipers, E. A. J. F. Peters, and J. T. Padding, J. Colloid Interface Sci. 510, 262 (2018).

[24] S. De, S. P. Koesen, R. V. Maitri, M. Golombok, J. T. Padding, and J. F. M. van Santvoort, AIChE J. 64, 773 (2018).

[25] S. De, J. van der Schaaf, N. G. Deen, J. A. M. Kuipers, E. A. J. F. Peters, and J. T. Padding, Phys. Fluids 29, 113102 (2017).

[26] D. F. James, R. Yip, and I. G. Currie, J. Rheol. 56, 1249 (2012).

[27] R. S. Seright, T. Fan, K. Wavrik, and P. Recovery, SPE 16, 24 (2011).

[28] See Supplemental Material at http://link.aps.org/supplemental/ 10.1103/PhysRevE.101.042605 for details of the movies and calculations.

[29] V. Schadler and E. J. Windhab, Desalination 189, 130 (2006).

[30] N. Aryanti and R. A. Williams, Part. Sci. Technol. 36, 913 (2018).

[31] R. H. Colby, Rheol. Acta 49, 425 (2010).

[32] K. S. Sorbie, Polymer Improved Oil Recovery (Blackie \& Son, Glasgow, 1991).

[33] J. Sheng, Modern Chemical Enhanced Oil Recovery: Theory and Practice (Gulf Professional Publishing, Burlington, MA, 2010).

[34] D. Weitz, H. Wyss, and R. Larsen, GIT Lab. J. 11, 68 (2007).

[35] P. G. De Gennes, J. Chem. Phys. 60, 5030 (1974).

[36] S. De, J. A. M. Kuipers, E. A. J. F. Peters, and J. T. Padding, Phys. Rev. Fluids 2, 053303 (2017).

[37] L. F. Lopes, B. M. Silveira, and R. B. Z L Moreno, Int. J. Eng. Technol. 14, 35 (2014).

[38] R. H. Colby and M. Rubinstein, Polymer Physics (Oxford University Press, Oxford, 2003).

[39] A. Samanta, A. Bera, K. Ojha, and A. Mandal, J. Chem. Eng. Data 55, 4315 (2010).

[40] S. Rellegadla, G. Prajapat, and A. Agrawal, Appl. Microbiol. Biotechnol. 101, 4387 (2017).

[41] C. M. Schroeder, H. P. Babcock, E. S. G. Shaqfeh, and S. Chu, Science 301, 1515 (2003).
[42] T. J. Ober, S. J. Haward, C. J. Pipe, J. Soulages, and G. H. McKinley, Rheol. Acta 52, 529 (2013).

[43] L. E. Rodd, T. P. Scott, D. V. Boger, J. J. Cooper-White, and G. H. McKinley, J. Non-Newtonian Fluid Mech. 129, 1 (2005).

[44] E. S. Boek, J. T. Padding, V. J. Anderson, W. J. Briels, and J. P. Crawshaw, J. Non-Newtonian Fluid Mech. 146, 11 (2007).

[45] S. J. Haward, T. J. Ober, M. S. N. Oliveira, M. A. Alves, and G. H. McKinley, Soft Matter 8, 536 (2012).

[46] W. Thielicke and E. J. Stamhuis, J. Open Res. Softw. 2, e30 (2014).

[47] G. Astarita, J. Non-Newtonian Fluid Mech. 6, 69 (1979).

[48] J. J. L. Higdon, Phys. Fluids A 5, 274 (1993).

[49] J. S. Lee, R. Dylla-Spears, N. P. Teclemariam, and S. J. Muller, Appl. Phys. Lett. 90, 074103 (2007).

[50] S. E. Mall-Gleissle, W. Gleissle, G. H. McKinley, and H. Buggisch, Rheol. Acta 41, 61 (2002).

[51] T. T. Perkins, Science 276, 2016 (1997).

[52] H. Bodiguel, J. Beaumont, A. Machado, L. Martinie, H. Kellay, and A. Colin, Phys. Rev. Lett. 114, 028302 (2015).

[53] M. Minale, Phys. Fluids 28, 023102 (2016).

[54] L. P. Paduano, T. Schweizer, C. Carotenuto, J. Vermant, and M. Minale, J. Rheol. 63, 569 (2019).

[55] N. Zamani, I. Bondino, R. Kaufmann, and A. Skauge, J. Pet. Sci. Eng. 133, 483 (2015).

[56] A. Keller and J. A. Odell, Colloid Polym. Sci. 263, 181 (1985).

[57] S. P. Carrington and J. A. Odell, Non-Newtonian Fluid Mech. 67, 269 (1996).

[58] P. Diamond, J. Harvey, J. Katz, D. Nelson, and P. Steinhardt, Drag Reduction by Polymer Additives (The MITRE corporation, McLean, Virginia, 1992).

[59] C. M. Schroeder, R. E. Teixeira, E. S. G. Shaqfeh, and S. Chu, Phys. Rev. Lett. 95, 018301 (2005).

[60] D. Kawale, P. E. Boukany, M. T. Kreutzer, W. R. Rossen, and P. L. J. Zitha, in Proceedings of the SPE Enhanced oil recovery conference, Kuala Lumpur, Malaysia, 2015 (2015) .

[61] J. G. Southwick and C. W. Manke, SPE Reserv. Eng. 3, 1 (1988).

[62] L. E. Rodd, J. J. Cooper-White, D. V. Boger, and G. H. McKinley, J. Non-Newtonian Fluid Mech. 143, 170 (2007).

[63] M. A. Alves and R. J. Poole, J. Non-Newtonian Fluid Mech. 144, 140 (2007). 